\title{
Manipulation of spin dephasing in InAs quantum wires
}

\author{
J. L. Cheng ${ }^{1,2}$, M. Q. Weng ${ }^{2}$, and M. W. $\mathrm{Wu}^{1,2} *$ \\ Structure Research Laboratory, University of Science ETechnology of China, Academia Sinica, Hefei, Anhui, 230026, China \\ Department of Physics, University of Science ETechnology of China, Hefei, Anhui, 230026, China ${ }^{\dagger}$
}

(November 5, 2018)

\begin{abstract}
The spin dephasing due to the Rashba spin-orbit coupling, especially its dependence on the direction of the electric field is studied in InAs quantum wire. We find that the spin dephasing is strongly affected by the angle of Rashba effective magnetic field and the applied magnetic field. The nonlinearity in spin dephasing time versus the direction of the electric field shows a potential revenue to manipulate the spin lifetime in spintronic device. Moreover, we figure out a quantity that can well represent the inhomogeneous broadening of the system which may help us to understand the many-body spin dephasing due to the Rashba effect.
\end{abstract}

PACS: 75.70.Ak, 73.40.Gk, 72.10.Di, 73.50.Bk

Since Datta and Das proposed a new type of electronic transistor that utilizes the electron spin freedom, ${ }^{1}$ many efforts have been devoted to the realization of the spintronic devices. ${ }^{2-4}$ As the function of these devices rely on the spin coherence, the key to realize these devices is to manipulate the spin coherence. The confined narrow band gap semiconductor systems such as quantum well and quantum wire are proposed to be good candidates for spintronic devices as the electron spin precession in these systems can be easily tuned by external gate voltages through the Rashba effect ${ }^{5}$ which states that the spin-orbit coupling is proportional to the applied and/or interface electric field. In quantum wells, the direction of electric field (DOEF) is always perpendicular to the interface. Therefore the manipulation of the spin coherence can only be realized through the magnitude of the electric field. While in quantum wire, both the direction and the magnitude of the electric field can be tuned by the external gate voltages. This provides one more degree of freedom to manipulate the spin coherence by alerting the DOEF. Here we demonstrate the possibility of using this additional degree of freedom to realize the manipulation of spin coherence in $n$-typed InAs quantum wire by calculating the dependence of the spin dephasing time (SDT) with the DOEF for different spin configurations.

It is known that for high temperature the spin dephasing magnetism for low dimensional $n$-typed InAs is due to the Rashba effect. ${ }^{5}$ We note here that the Rashba effect provides two spin dephasing channels: The first one is the effective spin-flip (SF) scattering caused by the anisotropy of the Rashba term and the spin conserving (SC) scattering. This dephasing effect has been widely discussed in the literature. The second one is a newly proposed many-body effect which is caused by the intrinsic inhomogeneous broadening (i.e. $k$-dependence) from the Rashba term itself together with the SC scattering.
Our previous works have shown that this many-body effect plays important, sometimes dominant, role in spin dephasing $^{6-10}$ as well as in spin transport. ${ }^{11,12}$ In this paper, we apply the many-body kinetic theory developed in our previous papers ${ }^{13,8-10}$ to study the spin dephasing in InAs quantum wire (QW) and show the feasibility of the manipulation of the spin coherence through changing the DOEF. Moreover, through changing the DOEF we are able to change the Rashba term and thus the inhomogeneous broadening alone while keeping the SC scattering unchanged. In this paper, we are going to have the inhomogeneous broadening quantified for the first time and show how the many-body spin dephasing depends on the inhomogeneous broadening.

The QW system we study is an InAs nano-structure confined by square potential wells with width $a$ in both perpendicular directions. In this system, the electron state is characterized by two subband indexes $n_{1}$ and $n_{2}$ which stand for the quantum number of two confined direction respectively, the $1 \mathrm{D}$ momentum $\mathbf{k}$ along the wire together with a spin index $\sigma$. In the present paper, we only consider the electron density that the subband separation is large enough so that only the lowest subband is populated and the transition to the upper subband is negligible. Therefore, we need only consider the subband with $n_{1}=n_{2}=1$, and drop the subband index consequently. In this study, the spin quantization direction is chosen to be $z$-axis. A moderate magnetic field is applied along the $x$-axis. Taking account of the Rashba effect, the electrons experience an additional wavevector dependent effective magnetic field

$$
\mathbf{h}(\mathbf{k})=\alpha_{0} \mathbf{E} \times \mathbf{k}
$$

where $\alpha_{0}$ is the Rashba spin-orbit parameter and $\mathbf{E}$ is the interface electric field. It is note that this electric field

*Email: mwwu@ustc.edu.cn

${ }^{\dagger}$ Mailing address. 
can be tuned by the gate voltages on both confined directions. Therefore, both the magnitude and the direction of this electric field can be tuned in the plane perpendicular to the wire. Under the two special configurations (see Fig.1) we study, the electric field and the Rashba effective magnetic field (REMF) are: (i) $\mathbf{E}=(E \sin \theta, E \cos \theta, 0)$, $\mathbf{h}(k)=\alpha E k(\cos \theta,-\sin \theta, 0)$ for the first configuration where the axis of QW is laid along $z$-direction; and (ii) $\mathbf{E}=(0, E \cos \theta, E \sin \theta), \mathbf{h}(k)=\alpha k E(0,-\sin \theta, \cos \theta)$ for the second configuration where the axis of QW is laid along the $x$-direction respectively. Here $\theta$ is the angle between the electric field and the $y$-axis. In configuration (i) it is also the angle between the REMF and the AMF. The interaction Hamiltonian $H_{I}$ is composed of Coulomb interaction $H_{e e}$, electron-phonon interaction $H_{p h}$, as well as electron-impurity scattering $H_{i}$. Their expressions can be found in textbooks. ${ }^{14,15}$

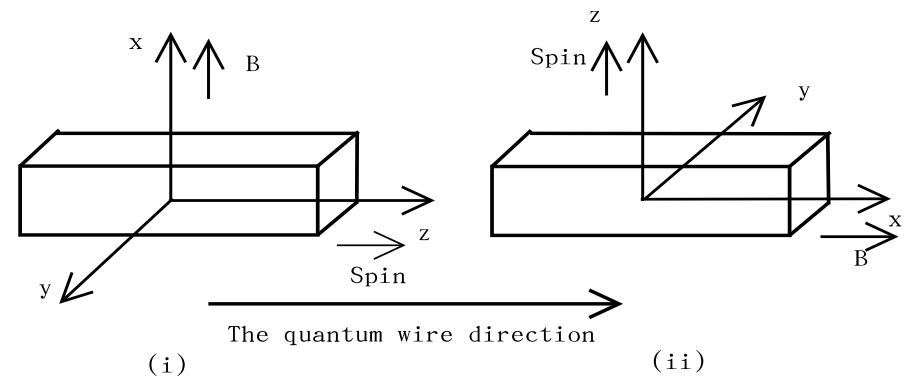

FIG. 1. The two special configurations we study. (i) the axis of the QW is laid along the $z$ direction; (ii) the axis of the QW is laid along the $x$ direction.

We construct the kinetic Bloch equations by the nonequilibrium Green function $\operatorname{method}^{14}$ as follows:

$$
\dot{\rho}_{\mathbf{k}, \sigma \sigma^{\prime}}=\left.\dot{\rho}_{\mathbf{k}, \sigma \sigma^{\prime}}\right|_{\mathrm{Coh}}+\left.\dot{\rho}_{\mathbf{k}, \sigma \sigma^{\prime}}\right|_{\text {Scatt }} .
$$

Here $\rho_{\mathbf{k}}$ represents the single particle density matrix. The diagonal elements describe the electron distribution functions $\rho_{\mathbf{k}, \sigma \sigma}=f_{\mathbf{k} \sigma}$. While the off-diagonal elements $\rho_{\mathbf{k}, \frac{1}{2}-\frac{1}{2}} \equiv \rho_{\mathbf{k}}$ are the inter-spin-band polarizations (coherence) of the spin coherence. ${ }^{16}$ Note that $\rho_{\mathbf{k},-\frac{1}{2} \frac{1}{2}} \equiv$ $\rho_{\mathbf{k}, \frac{1}{2}-\frac{1}{2}}^{*}=\rho_{\mathbf{k}}^{*}$.

The coherent part of the kinetic equations for the electron distribution function and the spin coherence are

$$
\left.\dot{f}_{k, \sigma}\right|_{c o h}=-2 \sigma \operatorname{Im}\left[\rho_{k}\left(R_{k, \downarrow \uparrow}-\frac{1}{2} g \mu_{B} B-\sum_{q} V_{q} \rho_{k-q}^{\star}\right)\right]
$$

and

$$
\begin{aligned}
\left.\dot{\rho}_{k}\right|_{c o h} & =-i\left[\left(R_{k, \uparrow \uparrow}-R_{k, \downarrow \downarrow}-\sum_{q} V_{q}\left(f_{k-q, \uparrow}-f_{k-q, \downarrow}\right)\right) \rho_{k}\right. \\
& +\left(R_{k, \uparrow \downarrow}-\frac{1}{2} g \mu_{B} B-\sum_{q} V_{q} \rho_{k-q}\left(f_{k \uparrow}-f_{k \downarrow}\right)\right]
\end{aligned}
$$

In these equations, the terms with $R_{k, \sigma \sigma^{\prime}}$ come from the Rashba term, and $R_{k, \sigma \sigma^{\prime}}=-\alpha_{0} E k \delta_{\sigma-\sigma^{\prime}}\{\sin \theta+i \sigma \cos \theta\}$ for configuration (i) and $R_{k, \sigma \sigma^{\prime}}=\alpha_{0} E k\left\{\delta_{\sigma \sigma^{\prime}} \cos \theta+\right.$ $\left.i \delta_{\sigma-\sigma^{\prime}} \sin \theta\right\}$ for configuration (ii). While the term with $V_{q}$ is the contribution of the electron-electron interaction up to $\mathrm{HF}$ approximation with $V_{q}$ denotes the 1D Coulomb matrix element under static screening. The scattering term $\left.\frac{\partial f_{k \sigma}}{\partial t}\right|_{\text {scatt }}$ and $\left.\frac{\partial \rho_{k}}{\partial t}\right|_{\text {scatt }}$ are listed detail in Refs. 8-10, and will not be repeated here.

The initial conditions at $t=0$ are taken as:

$$
\begin{aligned}
& \left.\rho_{\mathbf{k}}\right|_{\mathrm{t}=0}=0 \\
& \left.f_{\mathbf{k} \sigma}\right|_{\mathrm{t}=0}=1 /\left\{\exp \left[\left(\varepsilon_{\mathbf{k}}-\mu_{\sigma}\right) / k_{B} T\right]+1\right\}
\end{aligned}
$$

where $\mu_{\sigma}$ is the chemical potential for spin $\sigma$. The condition $\mu_{\frac{1}{2}} \neq \mu_{-\frac{1}{2}}$ gives rise to the imbalance of the electron densities of the two spin bands. Eqs. (2) through (4) together with the initial conditions Eqs. (5) and (6) comprise the complete set of kinetic Bloch equations of our investigation.

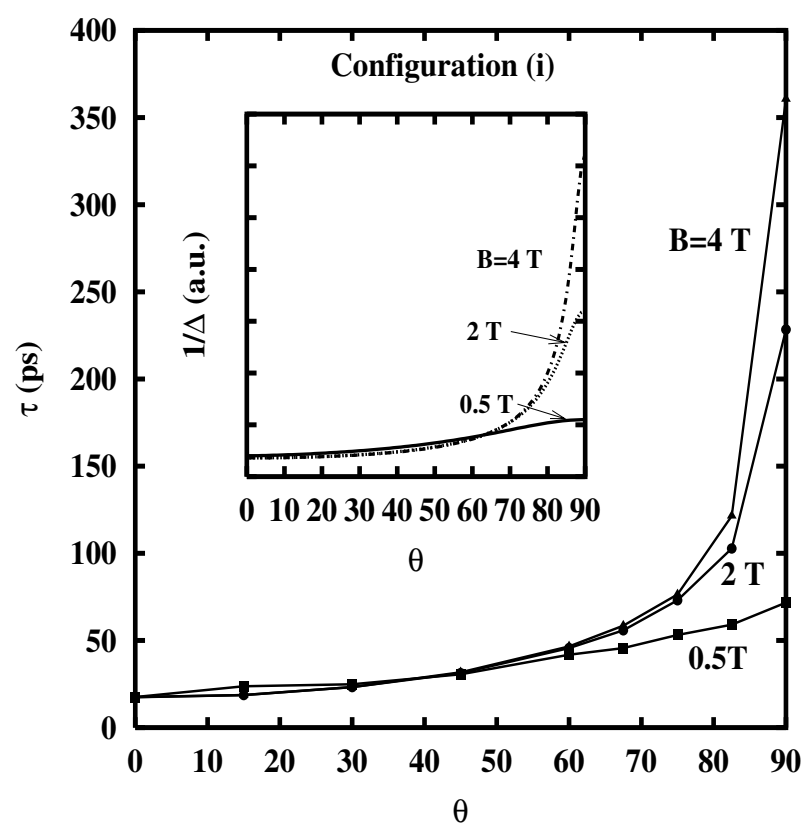

FIG. 2. The spin dephasing time vs. the angle of the interface electric field and $y$-axis for configuration (i) shown in Fig. 1 under different magnetic field: $(\boldsymbol{\square}) \mathrm{B}=0.5 \mathrm{~T} ;(\bullet) \mathrm{B}=2 \mathrm{~T}$; $(\boldsymbol{\Delta})$ and $\mathrm{B}=4 \mathrm{~T}$. The inset shows the standard deviation of the magnetic field the electron surfers.

By numerically solving the kinetic Bloch Eqs (2) and the initial condition (5) and (6), we are able to get the temporal evolution of the electron distribution function and the spin coherence self-consistently. As discussed in the previous papers, ${ }^{16,17}$ irreversible spin dephasing can be well defined by the slope of the envelope of the incoherently summed spin coherence $\rho(t)=\sum_{\mathbf{k}}\left|\rho_{\mathbf{k}}\right|$. In this way, we obtain the SDT which is defined as the inverse of the decay rate of the envelope of $\rho(t)$.

We calculate the SDT in an InAs QW under two config- 
urations shown in Fig. 1 at temperature $T=150 \mathrm{~K}$. With this relative high temperature we only need to consider the electron-longitudinal optical phonon, the electronimpurity and the electron-electron Coulomb scattering. In the calculation the interface electric field $E$, the density of the electron $N_{e}$, the well widths of the QW $a$ and the density of the impurities are chosen to be $1.4 \times 10^{4} \mathrm{~V} / \mathrm{cm}, 5.3 \times 10^{16} \mathrm{~cm}^{-3}, 15 \mathrm{~nm}$ and 0 respectively. The Rashba spin-orbit parameter $\alpha_{0}$ is $110 \AA^{218}$, and the other material parameters are cited from Ref. 19. By changing the direction of the electron field we are able to change the direction of the REMF. For configuration (i), by changing the DOEF, the angle between the AMF and REMF can be changed but both the AMF and the REMF are perpendicular to the spin polarization. While for configuration (ii), when the direction of the DOEF changes, the REMF is always normal to the AMF but the angle between the REMF and the spin polarization changes. With the different relative directions between the AMF, the REMF and the spin polarization, one may expect that the manipulation of the spin dephasing via the DOEF can be quite different under different configurations.

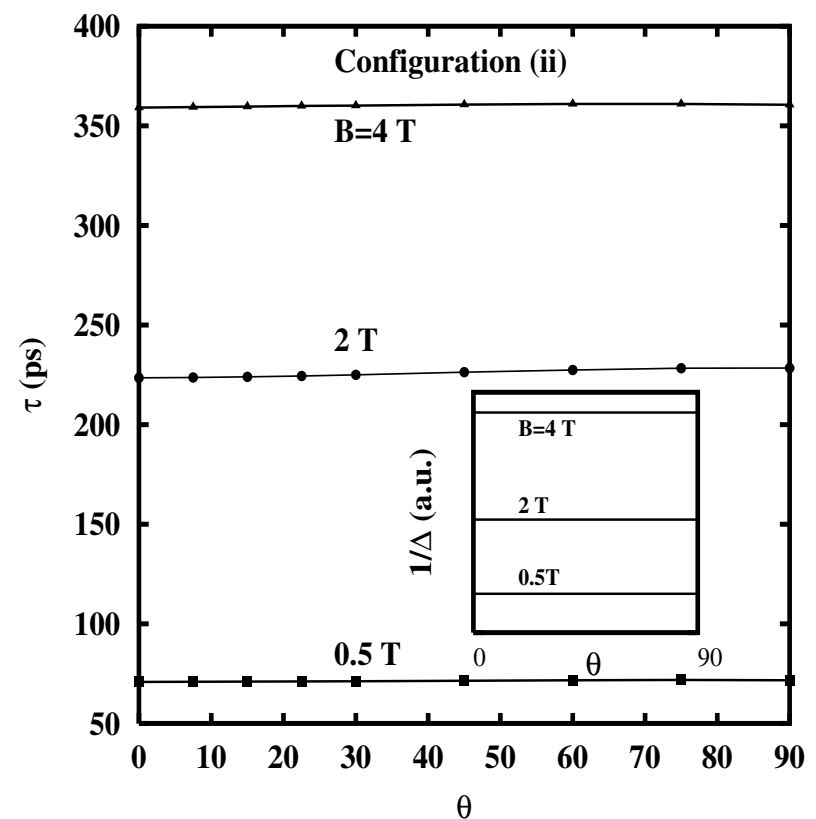

FIG. 3. The spin dephasing time vs. the angle of the interface electric field and $y$-axis for configuration (ii) shown in Fig. 1 under different magnetic field: $(\boldsymbol{\square}) \mathrm{B}=0.5 \mathrm{~T} ;(\bullet) \mathrm{B}=2 \mathrm{~T}$; $(\boldsymbol{\Delta})$ and $\mathrm{B}=4 \mathrm{~T}$. The inset shows the standard deviation of the magnetic field the electron surfers.

Our results are plotted as functions of the angle $\theta$ under different AMF's in Figs. 2 and 3 for the two special configurations. One notices that for configuration (i) when the REMF is parallel to the AMF, i.e. $\theta=0$, the SDT is insensitive to the magnetic field. Moreover, the SDT is also insensitive to $\theta$ when it is smaller than $60^{\circ}$. After that, SDT gets a rapid increase as $\theta$ increases for all of the AMF we study. For example when $B=2 \mathrm{~T}$, the SDT at $\theta=90^{\circ}$ is 3 times higher than that at $\theta=60^{\circ}$. The larger the AMF, the faster the SDT increases with $\theta$. As a result, the SDT increases with the AMF when the REMF is near the normal of the AMF. However, the result of the configuration (ii) is quite different: Here the SDT is independent on the DOEF under a moderate AMF. Nevertheless, it is noted that the SDT also increases with the $\mathrm{AMF}$ and the value is equal to the corresponding one in configuration (i) when the REMF is normal to the spin polarization, ie., $\theta=90^{\circ}$.

The DOEF dependence of spin dephasing can be understood from the many-body point of view. Besides the single particle spin dephasing due to the effective SF scattering, Rashba effect also provides an additional many-body spin dephasing channel through its inherit inhomogeneous broadening. It has been pointed out before the inhomogeneous broadening together with the SC scattering provides an irreversible spin dephasing. In the present case, the inhomogeneous broadening can be calculated as follows: The electrons in QW undergo the Larmor precession around the magnetic field composed of the AMF and the REMF. As the REMF is wavevector dependent, the frequency and the direction of the Larmor precession differs between electrons with different wavevectors. This is the origin of the inhomogeneous broadening. Therefore, one can expect that the inhomogeneous broadening of the frequency of Larmor precession may somehow reflect the spin dephasing due to the inhomogeneous broadening. Starting from this point, we carry out the corresponding standard deviation $\Delta$ of the Larmor frequencies:

$$
\begin{aligned}
\Delta^{2}= & \left\langle\left(g \mu_{B} B+\alpha_{0} E k \cos \phi\right)^{2}+\left(\alpha_{0} E k \sin \phi\right)^{2}\right\rangle \\
& -\left\langle\sqrt{\left(g \mu_{B} B+\alpha_{0} E k \cos \phi\right)^{2}+\left(\alpha_{0} E k \sin \phi\right)^{2}}\right\rangle^{2}
\end{aligned}
$$

with $\phi$ denoting the angle between $\mathbf{B}$ and $\mathbf{h}(\mathbf{k})$. In configuration (i), $\phi$ is $\theta$ and in configuration (ii) $\phi$ is always $90^{\circ} .\langle\cdots\rangle$ represents the average over the imbalance of the spin-up and spin-down electrons:

$$
\langle A(k)\rangle=\frac{\int d k\left(f_{k \uparrow}-f_{k \downarrow}\right) A(k)}{\int d k\left(f_{k \uparrow}-f_{k \downarrow}\right)} .
$$

In the inset of Figs. 2 and $3,1 / \Delta$ is plotted as a function of the $\phi(\theta)$ for two configurations. One can easily see that the $1 / \Delta-\theta$ curve is much similar to the $\tau-\theta$ curve: For configuration (i), $1 / \Delta$ is insensitive to the DOEF and the AMF when $\theta<60^{\circ}$. When $\theta>60^{\circ}, 1 / \Delta$ increases rapidly with both the $\mathrm{AMF}$ and $\theta$; For configuration (ii), $1 / \Delta$ does not depend on DOEF, and its value equals the corresponding one in configuration (i) with the REMF being perpendicular to the AMF.

From the correspondence between the $\tau-\theta$ curves and the $1 / \Delta-\theta$ curves one can conclude that the spin dephasing in the QW we study is determined by the inhomogeneous broadening. With the $1 / \Delta-\theta$ curve, it is easy to understand the AMF dependence and the DOEF dependence of the spin dephasing: For configuration (i), 
when the REMF is parallel to the AMF, i.e. $\theta=0, \Delta$ does not depend on the AMF. Therefore the AMF does not reduce the inhomogeneous broadening. As a consequence, the spin dephasing is insensitive to the magnetic field. Nonetheless when the REMF is perpendicular to the AMF, that is $\theta=90^{\circ}, \Delta$ reduces with the AMF. Consequently, the inhomogeneous broadening, thus the spin dephasing, is reduced by the AMF. For configuration (ii), the REMF is perpendicular to the AMF for all directions of the electric field. Therefore $\Delta$, the inhomogeneous broadening of the Larmor precession frequency, is independent of the DOEF and its value is the same to the corresponding one in configuration (i) when the $\theta=90^{\circ}$. As a result, the SDT in configuration (ii) does not change with $\theta$ and its value equals the corresponding value in configuration (i) when $\theta$ is $90^{\circ}$.

The nonlinear property in $\tau-\theta$ curve of configuration (i) can also be understood from the inhomogeneous broadening: When the AMF is much larger than the REMF, $\Delta$ is expected to be

$$
\begin{aligned}
& \Delta^{2}=\left\langle\left(\alpha_{0} E k\right)^{2}\right\rangle \cos ^{2} \theta+\frac{1}{4\left(g \mu_{B} B\right)^{2}}\left\{\left\langle\left(\alpha_{0} E k\right)^{4}\right\rangle\right. \\
& \left.\times\left[1-6 \cos ^{2} \theta+5 \cos ^{4} \theta\right]-\left\langle\left(\alpha_{0} E k\right)^{2}\right\rangle^{2} \sin ^{4} \theta\right\} .
\end{aligned}
$$

As the second term is very small, $1 / \Delta$ changes with $\theta$ according to $1 / \cos \theta$ except in the regime where $\theta$ is very close to $90^{\circ}$. Consequently, the spin dephasing changes slowly with $\theta$ when $\theta$ is smaller than $60^{\circ}$ and then gets a rapid increase after $60^{\circ}$.

In conclusion we have performed a kinetic study of the spin dephasing in InAs QW. We find that under a moderate magnetic field, the SDT in some configuration depends on the DOEF; while in the other configuration, it does not change with the DOEF. Therefore, for the right configuration, the spin dephasing can be tuned through changing the direction of the interface electric field in addition to the magnitude of the electric field. Moreover, we define a quality that well represents spin dephasing due to the inhomogeneous broadening of the Rashba term and show that the spin dephasing in the system we study is determined by the inhomogeneous broadening.

\section{ACKNOWLEDGMENTS}

MWW is supported by the "100 Person Project" of Chinese Academy of Sciences and Natural Science Foundation of China under Grant No. 10247002.

${ }^{1}$ S. Datta and B. Das, Appl. Phys. Lett 56, 665 (1990).

${ }^{2}$ M. Ziese and M. J. Thornton, Spin Electronics (Springer, Berlin, 2001).

${ }^{3}$ S. A. Wolf et al., Science 294, 1488 (2001).

${ }^{4}$ D. D. Awschalom, N. Samarth, and D. Loss, Semiconductor Spintronics and Quantum Computation (Springer, Berlin, 2002).

${ }^{5}$ Y. A. Bychkov and E. Rashba, Sov. Phys. JETP Lett. 39, 78 (1984).

${ }^{6}$ M. W. Wu and C. Z. Ning, Eur. Phys. J. B. 18, 373 (2000).

${ }^{7}$ M. W. Wu, J. Supercond.: Incorping Novel Mechanism 14, 245 (2001), cond-mat/0109258.

${ }^{8}$ M. Q. Weng and M. W. Wu, cond-mat/0302330.

${ }^{9}$ M. Q. Weng and M. W. Wu, cond-mat/0210313.

${ }^{10} \mathrm{M}$. Q. Weng and M. W. Wu, cond-mat/0303169.

${ }^{11}$ M. Q. Weng and M. W. Wu, Phys. Rev. B 66, 235109 (2002).

12 M. Q. Weng and M. W. Wu, J. Appl. Phys. 93, 410 (2003).

${ }^{13} \mathrm{M}$. W. Wu and C. Z. Ning, phys. stat. sol. B 222, 523 (2000).

${ }^{14}$ Haug and A. P. Jauho, Quantum Kinetics in Transport and Optics of Semiconductors (Springer-Verlag, Berlin, 1996).

${ }^{15}$ G. D. Mahan, Many-particle Physics (Plenum, New York, 1981).

${ }^{16}$ M. W. Wu and H. Metiu, Phys. Rev. B 61, 2945 (2000).

17 T. Kuhn and F. Rossi, Phys. Rev. Lett. 69, 977 (1992).

${ }^{18}$ G. Lommer, F. Malcher, and U. Rössler, Phys. Rev. Lett. 60, 728 (1988).

19 O. Madelung, M. Schultz, and H. W. (eds.), Numerical Data and Functional Relationships in Science and Technology, Landolt-Börnstein, New Series (Springer-Verlag, Berlin, 1982), Vol. 17. 